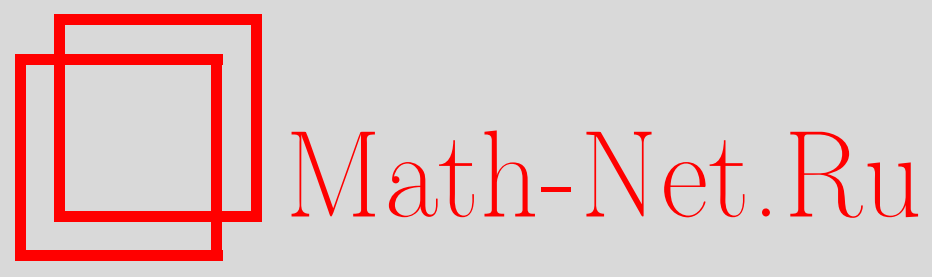

В. А. Емеличев, В. В. Коротков, О радиусе устойчивости эффективного решения многокритериальной задачи портфельной оптимизации с критериями Сэвиджа, Дискрет. матем., 2011, том 23, выпуск 4, 33-38

DOI: https://doi.org/10.4213/dm1159

Использование Общероссийского математического портала Math-Net.Ru подразумевает, что вы прочитали и согласны с пользовательским соглашением http://www . mathnet.ru/rus/agreement

Параметры загрузки:

IP : 54.166 .219 .16

26 апреля 2023 г., $11: 50: 26$

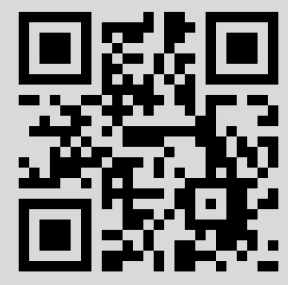




\title{
О радиусе устойчивости эффективного решения многокритериальной задачи портфельной оптимизации с критериями Сэвиджа
}

\author{
() 2011 г. В. А. Емеличев, В. В. Коротков
}

\begin{abstract}
На основе портфельной теории Марковица строится векторная инвестиционная булева модель с минимаксным критерием риска Сэвиджа. Получены нижняя и верхняя оценки радиуса устойчивости оптимального по Парето портфеля сформулированной модели, которые в частном случае превращаются в формулу радиуса устойчивости эффективного решения векторной линейной булевой задачи при соответствующем сочетании метрик в пространствах решений и критериев.
\end{abstract}

\section{1. Введение}

Вопросы устойчивости скалярных и векторных задач дискретной оптимизации в последние годы привлекают внимание многих специалистов (см., например, монографии [1-3] и обзоры [4-6], а также библиографии [7, 8]. Это в значительной степени связано с необходимостью учета неточности и некорректности исходной информации, которые свойственны реальным ситуациям решения задач. Основной вопрос, который при этом возникает, в каких пределах можно варьировать (возмущать) исходные данные задачи, чтобы найденное решение было устойчиво, то есть оставалось оптимальным (в векторном случае, например, оптимальным по Парето). Такая постановка вопроса приводит к понятию радиуса устойчивости решения как предельного уровня таких возмущений. Конкретное содержание этого понятия зависит от выбора множества параметров задачи, подверженных возмущениям, и от структуры, определяющей отношения близости на множестве исходных данных, то есть, от метрик, заданных в пространствах параметров. Большинство результатов в этом направлении связано с получением формул или оценок радиуса устойчивости эффективных (оптимальных по Парето) решений векторных задач дискретной оптимизации с линейными критериями [9-13]. В настоящей статье получены нижняя и верхняя оценки такого радиуса для многокритериальной булевой задачи с нелинейными критериями, а именно, для задачи портфельной оптимизации с минимаксными критериями риска Сэвиджа [14]. При этом в частном случае, когда критерии Сэвиджа становятся линейными, обе оценки превращаются в ранее известную формулу (см. [11]) радиуса устойчивости эффективного решения векторной линейной булевой задачи. Этого удалось добиться благодаря удачно подобранной комбинации октаэдральной $l_{1}$ и чебышевской $l_{\infty}$ метрик в трех пространствах параметров рассматриваемой инвестиционной задачи.

2 Дискретная математика, т.23 №4 
Для того, чтобы сформулировать задачу управления финансовыми инвестициями, основанную на известной портфельной теории Марковица [17], введем следующие обозначения:

$N_{n}=\{1,2, \ldots, n\}-$ активы (акции, облигации предприятий, недвижимость и т. п.);

$N_{m}$ - экономические стратегии инвестора;

$R-$ трехиндексная матрица рисков (упущенных возможностей) размера $m \times n \times s$ с элементами $r_{i j k}$ из $\mathbf{R}$;

$r_{i j k}-$ риск, которому подвергается инвестор, выбирая стратегию $i \in N_{n}$ и актив $j \in N_{n}$ по критерию $k \in N_{n}$;

$x=\left(x_{1}, x_{2}, \ldots, x_{n}\right)^{T} \in X \subset\{0,1\}^{n}-$ портфель активов инвестора, где

$$
x_{j}= \begin{cases}1, & \text { если инвестор выбирает актив } j, \\ 0 & \text { в противном случае. }\end{cases}
$$

Наряду с трехиндексной матрицей $R=\left[r_{i j k}\right] \in \mathbf{R}^{m \times n \times s}$, будем использовать двумерные сечения $\mathbf{R}_{k} \in \mathbf{R}^{m \times n}, k \in N_{s}$, этой матрицы.

Пусть на множестве векторов (решений) $X,|X| \geqslant 2$, задана вектор-функция

$$
f(x, R)=\left(f_{1}\left(x, R_{1}\right), f_{2}\left(x, R_{2}\right), \ldots, f_{s}\left(x, R_{s}\right)\right)
$$

с критериями минимального риска (крайнего пессимизма) Сэвиджа

$$
f_{k}\left(x, R_{k}\right)=\max _{i \in N_{n}} \sum_{j \in N_{n}} r_{i j k} x_{j} \underset{x \in X}{\longrightarrow} \min , \quad k \in N_{s} .
$$

Под векторной ( $s$-критериальной) задачей портфельной оптимизации $Z^{s}(R), s \geqslant 1$, будем понимать задачу поиска множества Парето $P^{s}(R)$, состоящего из эффективных портфелей

$$
P^{s}(R)=\left\{x \in X: P^{s}(x, R)=0\right\},
$$

где

$$
P^{s}(x, R)=\left\{x^{\prime} \in X: x \succ_{R} x^{\prime}\right\},
$$

а символ $\succ_{R}$ означает отношение, задаваемое на множестве $X$ формулой

$$
x \underset{R}{\succ} x^{\prime} \Longleftrightarrow g\left(x, x^{\prime}, R\right) \geqslant 0 \& g\left(x, x^{\prime}, r\right) \neq \mathbf{0},
$$

где

$$
\begin{aligned}
\mathbf{0} & =(0,0, \ldots, 0) \in \mathbf{R}^{s}, \\
g\left(x, x^{\prime}, R_{k}\right) & =\left(g_{1}\left(x, x^{\prime}, r_{1}\right), g_{2}\left(x, x^{\prime}, R_{2}\right), \ldots, g_{s}\left(x, x^{\prime}, R_{s}\right)\right), \\
g_{k}\left(x, x^{\prime}, R_{k}\right) & =f_{k}\left(x, R_{k}\right)-f_{k}\left(x^{\prime}, R_{k}\right) \\
& =\max _{i \in N_{m}} R_{i k} x-\max _{i \in N_{m}} R_{i k} x^{\prime} \\
& =\min _{i^{\prime} \in N_{m}} \max _{i \in N_{m}}\left(R_{i k} x-R_{i^{\prime} k} x^{\prime}\right), \quad k \in N_{s},
\end{aligned}
$$


a $R_{i k}=\left(r_{i 1 k}, r_{i 2 k}, \ldots, r_{i n k}\right)-i$-я строка сечения $R_{r} \in \mathbf{R}^{m \times n}, i \in N_{m}$.

$\mathrm{B}$ пространствах решений $\mathbf{R}^{n}$ и стратегий $\mathbf{R}^{m}$ зададим метрику $l_{1}$, а в критериальном пространстве $\mathbf{R}^{s}-$ метрику $l_{\infty}$, то есть полагаем

$$
\begin{array}{rlrl}
\left\|R_{i k}\right\|_{1} & =\sum_{j \in N_{n}}\left\|r_{i j k}\right\|, & i \in N_{m}, \quad k \in N_{s}, \\
\left\|R_{k}\right\|_{1} & =\sum_{i \in N_{m}}\left\|R_{i k}\right\|_{1}=\sum_{i \in N_{m}} \sum_{j \in N_{n}}\left|r_{i j k}\right|, & k \in N_{s}, \\
\|R\|_{\infty} & =\max _{k \in N_{S}}\left\|R_{k}\right\|_{1}=\max _{k \in N_{s}} \sum_{i \in N_{m}}\left\|R_{i k}\right\|_{1} \\
& =\max _{k \in N_{S}} \sum_{i \in N_{s}} \sum_{j \in N_{n}}\left|r_{i j k}\right| .
\end{array}
$$

В принятых обозначениях очевидны неравенства

$$
\left\|R_{i k}\right\|_{1} \leqslant\left\|R_{k}\right\|_{1} \leqslant\|R\|_{\infty}, \quad i \in N_{m}, \quad k \in N_{s},
$$

и

$$
\begin{aligned}
R_{i k} x-R_{i^{0} k} x^{0}-R_{i^{0} k} x^{0} & \geqslant-\left\|R_{i k}\right\|_{1}-\left\|R i^{0} k\right\|_{1} \\
& \geqslant-\left\|R_{k}\right\|_{1}, \quad i, i^{0} \in N-m, \quad k \in N_{s},
\end{aligned}
$$

для любой матрицы $R_{r} \in \mathbf{R}^{m \times n}$ и любых векторов $x^{0}, x \in X$.

Как обычно [9-12], радиусом устойчивости портфеля $x^{0} \in P^{s}(R)$ назовем число

$$
\rho^{s}\left(x^{0}, R\right)= \begin{cases}\sup \Xi, & \text { если } \Xi \neq \varnothing, \\ 0, & \text { если } \Xi=\varnothing,\end{cases}
$$

где

$$
\begin{aligned}
& \Xi=\left\{\varepsilon>0: \forall R^{\prime} \in \Omega(\varepsilon), x^{0} \in P^{s}\left(R+R^{\prime}\right)\right\}, \\
& \Omega=\left\{R^{\prime} \in \mathbf{R}^{m \times n \times s}:\left\|R^{\prime}\right\|_{\infty}<\varepsilon\right\}
\end{aligned}
$$

- множество возмущающих матриц, $P^{s}\left(R+R^{\prime}\right)-$ множество Парето возмущенной задачи $Z^{s}\left(R+R^{\prime}\right)$.

Тем самым, радиус устойчивости задает предельный уровень возмущений исходных данных задачи (элементов матрицы $R$ ), которые сохраняют эффективность портфеля.

Очевидно следующее утверждение.

Лемма 1. Пусть $X^{0} \in P^{s}(R), \gamma>0$. Если для каждой возмущчающчейматрищьы $R^{\prime} \in \Omega(\gamma)$ и любого вектора $x \in X \backslash\left\{x_{0}\right\}$ найдется такой индекс $q \in N_{s}$, что справедливо неравенство $g_{q}\left(x, x^{0}, R_{q}+R_{q}^{\prime}\right)>0$, то портфель $x^{0}$ является эффективным решением возмущенной задачи $Z^{s}\left(R+R^{\prime}\right)$, то есть $x^{0} \in P^{s}\left(R+R^{\prime}\right)$ при $R^{\prime} \in \Omega(\gamma)$.

Теорема 1. Пусть в пространствах решений $R^{n}$ и стратегий $\mathbf{R}^{m}$ задана метрика $l_{1}, a$ в критериальном пространстве $\mathbf{R}^{s}$ метрика $l_{\infty}$. Тогда при

$$
\varphi=\min _{x \in X \backslash\left\{x^{0}\right\}} \max _{k \in N_{s}} \min _{i^{0} \in N_{m}} \max _{i \in N_{m}}\left(R_{i k} x-R_{i} k_{k} x^{0}\right)^{\prime}
$$

справедливы следующие оцуенки для радиуса устойчивости $\rho^{s}\left(x^{0}, R\right)$ эффективного портфеля $x^{0} \in P^{s}(R)$ :

$$
\varphi \leqslant \rho^{s}\left(x^{0}, R\right) \leqslant m \varphi .
$$


Доказательство. Пусть $x^{0} \in P^{s}(R)$. Очевидно, что $x \notin R\left(x^{0}, R\right)$, если $x \in X \backslash\left\{x^{0}\right\}$. Поэтому $\varphi \geqslant 0$.

Сначала докажем, что справедливо неравенство $\rho^{s}\left(x^{0}, R\right) \geqslant \varphi$, которое очевидно при $\varphi=0$. Согласно определению числа $\varphi$, для любого вектора $x \in X \backslash\left\{x^{0}\right\}$ существует такой индекс $q=q(x) \in N_{s}$, что

$$
\min _{i^{0} \in N_{m}} \max _{i \in N-m}\left(R_{i q} x-R_{i^{0} q} x^{0}\right) \geqslant \varphi .
$$

Далее, учитывая (1), получаем, что для любой матрицы $R^{\prime} \in \mathbf{R}^{m \times n \times s}$ и всякого индекса $k \in N_{s}$ справедливы равенства

$$
\begin{aligned}
g_{k}\left(x, x^{0}, R_{k}+R_{k}^{\prime}\right) & =\max _{i \in N_{m}}\left(R_{i k}+R_{i k}^{\prime}\right) x-\max _{i \in N_{m}}\left(R_{i k}+R_{i k}^{\prime}\right) x^{0} \\
& =\min _{i^{0} \in N_{m}} \max _{i \in N_{m}}\left(R_{i k} x-R_{i^{0} k} x+R_{i^{0} k}^{\prime} x-R_{i^{0} k}^{\prime} x^{0}\right) \\
& \geqslant \min _{i^{0} \in N_{m}} \max _{i \in N_{m}}\left(R_{i k}-R_{i^{0} k} x^{0}\right)=\left\|R_{k}^{\prime}\right\|_{1} .
\end{aligned}
$$

Отсюда, полагая $R^{\prime} \in \Omega(\varphi)$, ввиду (2), получаем, что

$$
g_{q}\left(x, x^{0}, R_{q}+R_{q}^{\prime}\right) \geqslant \varphi-\left\|R_{q}^{\prime}\right\|_{1} \geqslant \varphi-\left\|r_{q}^{\prime}\right\|_{\infty}>0 .
$$

Поэтому, в силу леммы $1, x^{0} \in P^{s}(R+R /)$ при любой возмущающей матрице $R^{\prime} \in \Omega(\varphi)$.

Наконец, докажем неравенство $\rho^{s}\left(x^{0}, R\right) \leqslant m \varphi$. Согласно определению числа $\varphi$, существует такой вектор $\hat{x} \in X \backslash\left\{x^{0}\right\}$, что справедливы неравенства

$$
\left.g_{k}\left(\hat{x}, x^{0}, R\right) k\right) \leqslant \varphi, \quad f \in N_{s} .
$$

Так как $\hat{x} \neq x^{0}$, найдется такой индекс $l \in N_{n}$, что $\hat{x} \neq x_{l}^{0}$. Полагая $\varepsilon>m \varphi$, рассмотрим возмущающую матрицу ${ }^{0}=\left[r_{i j k}^{0}\right] \in \mathbf{R}^{m \times n \times s}$, элементы которой определим следующим образом:

$$
r_{i j k}^{0}= \begin{cases}-\delta \operatorname{sign}\left(\hat{x}_{j}-x_{j}^{0}\right), & \text { если } i \in N_{m}, j=l, k=N_{s} \\ 0, & \text { если } i \in N_{m}, j \in N_{n} \backslash\{l\}, k \in N_{s},\end{cases}
$$

где $\varphi<\delta<\varepsilon / m$. Тогда

$$
\left\|R^{0}\right\|_{\infty}=\left\|R_{k}^{0}\right\|_{1}=m\left\|R_{i k}^{0}\right\|_{1}=m \delta
$$

при $i \in N_{m}, k \in N_{s}$, то есть $R^{0} \in \Omega(\varepsilon)$. Кроме того, все строки $R_{i k}^{0}, i \in N_{m}$, сечения $R_{k}^{0}$ одинаковы при любом индексе $k \in N_{s}$. Поэтому, обозначив такую строку через $B$ (зависит лишь от $\hat{x}$ и $x^{0}$ ), получаем, что

$$
B\left(\hat{x}-x^{0}\right)=-\delta, \quad\|B\|_{1}=\delta .
$$

Отсюда для любого индекса $k \in N_{s}$ в силу (3) следует, что

$$
\begin{aligned}
g_{k}\left(\hat{x}, x^{0}, R_{k}+R_{k}^{0}\right) & =\max _{i \in N_{m}}\left(R_{i k}+B\right) \hat{x}-\max _{i \in N_{m}}\left(R_{i k}+B\right) x^{0} \\
& =g_{k}\left(\hat{x}, x^{0}, R_{k}\right)+B\left(\hat{x}-x^{0}\right) \leqslant \varphi-\delta<0 .
\end{aligned}
$$


Таким образом, верно бинарное отношение $x^{0} \underset{R+R^{0}}{\succ} \hat{x}$. Следовательно, для любого числа $\varepsilon>m \varphi$ существует такая возмущенная матрица $R^{0} \in \Omega(\varepsilon)$, что эффективный портфель $x^{0}$ задачи $Z^{s}(R)$ перестает быть эффективным в возмущенной задаче $z^{s}\left(R+R^{0}\right)$, то есть $x^{0} \notin P^{s}\left(R+R^{0}\right)$. Поэтому $\rho^{s}\left(x^{0}, R\right) \leqslant m \varphi$.

Теорема доказана.

Отметим, что в [16] анонсированы аналогичные оценки (снизу и сверху) радиуса квазиустойчивости задачи $Z^{s}(R)$ в случае, когда во всех пространствах $\mathbf{R}^{m}, \mathbf{R}^{n}$ и $\mathbf{R}^{s}$ задана одна и та же чебышевская метрика $l_{\infty}$.

Обе оценки радиуса устойчивости $\rho^{s}\left(x^{0}, R\right)$, указанные в теореме, являются достижимыми при $m=1$. Действительно, пусть $m=1$. Тогда наша задача $Z^{s}(R)$ превращается в векторную ( $s$-критериальную) задачу булева программирования с линейными критериями

$$
R_{k} x \underset{x \in X}{\longrightarrow} \min , \quad k \in N_{s},
$$

где $R_{k}-k$-я строка матрицы $R \in \mathbf{R}^{s \times n}$. Поэтому из теоремы вытекает следующий известный результат.

Следствие 1 ([11]). Если в пространстве решений задана метрика $l_{1}$, а в критериальном пространстве метрика $l_{\infty}$, то для радиуса устойчивости эффективного решения $x^{0}$ векторной линейной булевой задачи (4) справедлива формула

$$
\rho^{s}\left(x^{0}, R\right)=\min _{x \in X \backslash\left\{x_{0}\right\}}\left\|\left(R\left(x-x^{0}\right)\right]^{+}\right\|_{\infty} .
$$

Здесь $\left(a^{+}\right)$- положительная срезка вектора $a=\left(a_{1}, a_{2}, \ldots, a_{s}\right)^{T}$, то есть

$$
(a)^{+}=\left(a_{1}^{+}, a_{2}^{+}, \ldots, a_{s}^{+}\right), \quad a_{i}^{+}=\max \left\{0, a_{i}\right\}, \quad i=1, \ldots, s .
$$

Эффективный портфель $x^{0} \in P^{s}(R)$ задачи $Z^{s}(R)$ назовем устойчивым, если $\rho^{s}\left(x^{0}, R\right)>0$. Кроме того, портфель $x^{0} \in X$ назовем строго эффективным (оптимальным по Смейлу [17]), если среди элементов множества $X \backslash\left\{x^{0}\right\}$ не существует такого решения $x$, что $f(x, R) \leqslant f\left(x^{0}, R\right)$.

Следующий критерий с очевидностью вытекает из теоремы 1.

Следствие 2. Эффективный портфель $x^{0} \in P^{s}(R)$ задачи $Z^{s}(R)$ устойчив в том и только в том случае, когда он строго эффективен.

\section{Список литературы}

1. Сергиенко И. В., Козерацкая Л. Н., Лебедева Т. Т., Исследование устойчивости и параметрический анализ дискретных оптимизационных задач. Наукова думка, Киев, 1995.

2. Сергиенко Т. И., Шило В. П., Задачи дискретной оптимизации. Наукова думка, Киев, 2003.

3. Сотсков Ю. Н., Сотскова Н. Ю., Теория расписаний. Системы с неопределенными числовыми параметрами. ОИПИ НАН Беларуси, Минск, 2004.

4. Sotskov Yu. N., Leontev V. K., Gordeev E. N., Some concepts of stability analysis in combinatorial optimization. Discrete Appl. Math. (1995) 58, 169-190.

5. Sotskov Yu. N., Tanaev V. S., Werner F., Stability radius of an optimal schedule: A survey and recent developments. Appl. Optim. (1998) 16, 72-108. 
6. Emelichev V. A., Girlich E., Nikulin Yu. V., Podkopaev D. P., Stability and regularization of vector problems of integer linear programming. Optimization (2002) 51, 645-676.

7. Greenberg H. J., A bibliography for the development of an intelligent mathematical programming system. Ann. Oper. Res. (1996) 65, 55-90.

8. Greenberg H. J., An annotated bibliography for post-solution analysis in mixed integer programming and combinatorial optimization. In: Advances in Computational and Stochastic Optimization, Logic Programming, and Heuristic Search. Interfaces in Computer Science and Operations Research (Woodruff D. L., ed.). Kluwer, Boston, 1998, pp. 97-147.

9. Емеличев В. А., Похилько В. Г., Анализ чувствительности эффективных решений векторной задачи минимизации линейных форм на множестве подстановок. Дискретная математика (2000) 12, №3, 37-48.

10. Емеличев В. А. Степанишина Ю. В., Многокритериальные комбинаторные линейные задачи: параметризация принципа оптимальности и устойчивость эффективных решений. Дискретная математика (2001) 13, №4, 43-51.

11. Емеличев В. А., Кузьмин К. Г., Общий подход к исследованию устойчивости паретооптимального решения векторной задачи целочисленного линейного программирования. Дискретная математика (2007) 19, №3, 79-83.

12. Емеличев В. А., Карелкина О. В., Конечные коалиционные игры: параметризация концепции равновесия (от Парето до Нэша) и устойчивость эффективной ситуации в метрике Гельдера. Дискретная математика (2009) 21, №2, 43-59.

13. Emelichev V., Podkopaev D., Quantitative stability analysis for vector problems of $0-1$ programming. Discrete Optim. (2010) 7, 48-63.

14. Savage L. J., The foundations of statistics. Dover, New York, 1972.

15. Markowitz H. M., Portfolio selection: efficient diversification of investments. Wiley, New York, 1959.

16. Емеличев В. А., Коротков В. В., О радиусе квазиустойчивости векторной булевой задачи с критерием Сэвиджа. В сб.: Материалы конф. «Дискретная оптимизащия и исследование операиций». Ин-т матем., Новосибирск, 2010, с. 113.

17. Smale S., Global analysis and economics: Pareto theory with constraints. J. Math. Econ. (1974) 1, 213-221.

Статья поступила 15.09.2010. 